\title{
Free and (Mostly) Open Source Data Analysis Software for Academic Research \\ Dr. Chinchu C.
}

\begin{abstract}
Data analysis is a crucial task in knowledge creation in social sciences. Free resources for data analysis provide researchers with greater freedom and make the research process more accessible and democratic. This article lists some free software which can perform basic and advanced statistical data analysis tasks. Some software which can perform other tasks such as text mining are also introduced. Ease of use and functionality are the major criteria for selecting these software packages.
\end{abstract}

Keywords: Free Software, Data Analysis, Social Science, Research 


\section{Free and (Mostly) Open Source Data Analysis Software for Academic Research \\ Dr. Chinchu C.}

\section{Introduction}

Research is a knowledge creation process and hence crucial to human progress. However there have been attempts to transform and treat knowledge as a commodity and use it for economic gain. This is generally viewed as an obstruction to the growth of research and to the propagation of knowledge. Research in social sciences generally requires the analysis of data in various forms. Use of software tools is an imperative in this situation. The range of software needed range from spreadsheets and statistical software to big data analysis and qualitative data analysis tools. Nonetheless the proprietary software tools available for use in social science research are priced so high that it is almost impossible for individuals or even small institutions to buy them. This may also lead to instances of piracy which is again a punishable offence and an unethical act, detrimental to the quality of research as well. It is in this context that the use of free and open-source (FOSS) software tools becomes a necessity and a political act for keeping knowledge free of restrictions. In a broader sense, use and promotion of free software is an act of responsibility towards the future generations. This paper presents an introduction to a number of free and/or open source software packages which can be used for various purposes, mainly by social science researchers.

\section{The Concept of Knowledge Freedom}

The idea that knowledge, in its various forms should be freely available to all, is not new. Foucault (1980) has maintained that knowledge is inextricably linked to power. Thus knowledge can be viewed as a form of power. In the academia too, knowledge acts as a form of soft power which can determine the prospects of one's career growth. From such a 
position, it becomes imperative that anyone who works for equality and egalitarianism should also work for making knowledge accessible to all.

The free software movement is based on the philosophy that considers software as a form of knowledge. The movement for software freedom was initiated because of the rampant commercialization in the field of software. Proprietary software, also known as licensed software, imposes a number of restrictions on the user, even after she/he has bought it. This typically includes restrictions on copying, sharing, modifying etc. The restraint on modifying the source code of software packages is also a hindrance to the learning process inherent in the process. Thus it can be argued that even after one buys a software, the ownership is not entirely transferred to the buyer.

Free and Open Source Software (FOSS) are different from proprietary software in that they give freedom to the user to run, change or modify, share, and copy a software. The term 'free' denotes freedom, not free-of-cost.

\section{Software Licenses}

Software packages are distributed under a variety of different licenses, each with a different view on the rights of the end-user of the product. The software are usually referred to with the type of license that they belong to. The license types that are relevant to research tools fall under any of the categories listed below.

Proprietary: The absolute ownership of the software rests with the provider, not with the customer. Usually the customer is given various rights to use the software, under an end-user license agreement (EULA). There will be restrictions on the copying, sharing, modifying or any such operations on the product. Some software need monthly or annual licensing with payments to the provider. 
Open Source: Open source software give the underlying programming code of the software to the user. The user may modify and even re distribute the modified version, under the terms of the license agreement. A number of license formats are used to distinguish between different agreements between the provider and the user.

Free Software: Free software may either be provided free of cost, or at a price. The term 'free' refers to liberty of usage, not price. Even though all free software are open-source, all open-source software may not be classified as free software, according to the philosophical issues existing between the two movements.

Apart from these, there exists other forms of licensing such as freewares, which are packages provided free of cost, but without the source code, and freemium packages, where a basic version of the software is provided freely and more advanced versions have to be purchased.

\section{Statistical analysis software}

A list of free or open-source software packages, mainly used for quantitative statistical analyses, is given here. Even though most of them provide support for advanced procedures like text mining, the primary usage is taken as criteria to include them under the category of statistical software. The list is not exhaustive, and is curated mostly based on individual preference and experience of the author.

R: Even though touted as a free alternative to proprietary statistical analysis packages including SPSS, $\mathrm{R}$ is not a software package in itself. Rather, $\mathrm{R}$ is a programming language and an environment for statistical analyses (Ihaka \& Gentleman, 1996). It is a dynamic environment, offering many facets for development (Chambers, 2009). R Being a programming language, $\mathrm{R}$ offers a steep learning curve for those without a basic training in programming. A number of Graphical User Interfaces (GUI) that use R in the background are 
available offering reliable and fast data analysis capabilities. This include R Commander, RKWard, Deducer, Rattle, Jamovi etc.

R Commander: R Commander, developed by John Fox, is a GUI for the R programming language. It offers one of the most powerful and user-friendly interfaces to use $\mathrm{R}$ for statistical data analysis (Downie, 2016). R Commander, along with its plug-ins like the EZR and FactoMineR provide a number of functionalities to perform a range of advanced statistical analyses. R Commander has to be installed as a package from within $\mathrm{R}$, and is not available as an executable installation file.

Deducer: Deducer is another GUI built as a potential replacement for proprietary data analysis software (Fellows, 2012). Unlike R Commander, Deducer, along with the JGR (Java GUI for R) console of R, can be downloaded and installed as a separate package. This makes installation easier. Once installed, Deducer offers an analysis experience that is much similar to SPSS, with its intuitive and familiar menu structure. Descriptive and inferential statistics are available as menu items similar to the popular proprietary packages.

PSPP: PSPP is a GNU project for statistical data analysis (GNU Project, 2015). It is sponsored by the Free Software Foundation. For users familiar with SPSS, PSPP offers an interface which is almost a clone of the SPSS menu system. It has a data/variable view window and menus labeled Data, Transform, Analyze, etc. all of which are reminiscent of SPSS. Unlike SPSS, PSPP can be downloaded and used free of cost, and offers a good portion of capabilities provided by the proprietary package. PSPP supports over one billion cases and variables, and is inter-operable with multiple software like LibreOffice and OpenOffice.Org. The data files and syntax are compatible with SPSS as well. Elaborate documentation is available and there is an active online global community that supports the users. 
Openstat: Openstat is a free package for statistical analysis developed by William Miller (Miller, 2013). Initially designed as an instructional package, it provides a large number of statistical operations to users, with detailed documentation. Openstat is designed for the Windows platform only, and may need compatibility software to run in Linux or Mac platforms.

RapidMiner: RapidMiner is a software platform, first developed by Ralf Klinkenberg, Ingo Mierswa, and Simon Fischer in 2001. It was initially named YALE (Yet Another Learning Environment) and later changed to RapidMiner in 2013. It is a freemium software, which means a low-key edition is available as free software and the full version is proprietary. RapidMiner is available for Windows, Linux and Mac platforms.

SOFA: SOFA (Statistics Open For All) is one of the most user-friendly and intuitive statistical packages currently available. The software, developed by Paton-Simpson \& Associates Ltd, can be run on Windows, Linux and Mac platforms (SOFA, 2017). A good collection of descriptive and inferential statistical tests, as well as visualizations are provided. The software adopts an instinctive approach where the user is guided through the various steps to be followed in the analysis, starting from choosing the procedures based on the type of data and results expected.

JASP: JASP is one of the most sought-after free and open-source alternatives to the proprietary packages. JASP was created by pooling resources from various Universities and research funds. In addition to the frequentist procedures offered by traditional software, JASP provides Bayesian inferential tests as well. Available in Windows, Linux, and Mac platforms, the tables and plots produced by JASP are in APA style, which helps in preparing manuscripts. The addition of Bayesian statistics gives an edge to JASP in terms of scientific rigour. (The JASP Team, 2015). 
Jamovi: As of 2021, Jamovi is probably the frontrunner in terms of ease of use and functionality among the free and open source packages which aim to provide a simple and powerful alternative to proprietary data analysis software such as SPSS and Stata (The jamovi project, 2020). Jamovi is an R based GUI which is easy to install and use, with most functions needed for an academic research project in social sciences bundled with the default installation. Besides, there is the option of adding more specialized modules using the jamovi library. These modules add a plethora of additional capabilities to jamovi. With continuous updates being released, what also make jamovi special is its ability to dynamically modify analysis results when the underlying data is modified. Cross-platform compatibility is also ensured, with the ability to import data in formats such as csv, sav (used by SPSS), dta (used by Stata) etc. Detailed documentation and third party tutorials are also available for jamovi. In India, Jamovi has become part of many workshops and training programmes in research methodology, where SPSS used to be the default choice.

MicrOsiris: Microsiris was developed at the University of Michigan. It is based on an earlier package, OSIRIS IV, developed at the same University. It is a data management and and statistical analysis tool. Speed and lightness are the distinct features of Microsiris. Microsiris offers a good user experience, with a clean interface and features like provision to deal with missing data. It can also import data from various platforms, including SPSS and SAS.

PAST: PAST (PAleontological STatistics) (Hammer et al., 2001) is another free software package which has recently been recognized as a viable alternative to proprietary software across social sciences. Originally developed in 2001 for use in Paleontology research, it has later been adapted to be used in various other fields. PAST offers a rich menu of statistical procedures to researchers, with some features like simple phylogenetic analysis exclusive for the paleontology and ecology fields. Features like Google Maps integration are unique to this package. Only Windows platform is supported at present. 
Gretl: Gretl is an open-source and multi-platform software package designed mainly for econometrics. It is considered as a free software alternative to the popular econometrics software, EViews, which is a proprietary product. Launched in 2000, gretl is an acronym for Gnu Regression, Econometrics and Time-series Library. A large range of menus are available for procedures like time series analysis. Apart from English, gretl supports more than 15 languages, including French, Spanish and Chinese.

Bluesky Statistics: Bluesky became an open source project in 2018, and is being developed by a community ever since. This is yet another R based GUI which aims to provide a pointand-click data analysis experience to social science researchers and students. It provides a familiar user interface for users who have used SPSS or similar software, and some elements, such as the ribbon below the menu bar resemble popular office packages. The desktop version of Bluesky is available for free download and use. Like modules in jamovi, extensions are available for Bluesky. There is a 'variable and data view' functionality similar to SPSS, and a number of basic and advanced data analysis options too. Books featuring Bluesky for advanced statistical applications have been published (Lamprianou, 2019).

SalStat: SalStat is another free data analysis software written in the Python language. Ease of use is a guiding principle for its development, and the developers have designed it particularly for researchers in social sciences, agriculture and biological sciences, business analytics, market research etc. Some feature like scraping data from web pages is also available in SalStat, making it more than just a data analysis tool. A number of descriptive analyses are available, and decent amount of inferential statistics are provided, including linear regression. SalStat does not support importing the sav file format used in SPSS. No new updates have been released since 2018 . 
Ministat Application: Ministat is an Android application which tries to provide a complete data analysis experience in a smartphone, and it does this job reasonable well. Ministat provides data visualizations, t-tests, ANOVA, correlation tests, regression analyses, and much more. It also provides some useful e-books which can be accessed only if one agrees to watch an advertisement. A premium version of the application without the advertisements is also available for those willing to pay. Though not much popular, with only $10 \mathrm{k}+$ downloads as of December 2021, Ministat provides a good experience of data analysis with a smartphone and is worth a try.

\section{Software for other research purposes}

The software packages in this list are either data mining packages or CAQDAS (Computer Aided Qualitative Data Analysis Software). Most of them are able to perform descriptive and inferential statistical operations as well.

Tanagra: Tanagra is a free data mining platform, intended for use in research and academics. It was developed as an experimental platform for students and researchers (Rakotomalala, 2005). It provides support for machine learning along with exploratory data analysis. The source code is provided for those interested in working on similar projects. Even though written as instructional software, Tanagra is widely used in actual studies as well. It has also been recommended for small-scale industrial usage (Enright \& Klippenstein, 2004).

Weka: Named after a flightless bird found only in the New Zealand, Weka (Waikato Environment for Knowledge Analysis) is a product of the University of Waikato, New Zealand. It is a multi-platform machine learning software written in Java. Weka is used for educational and research purposes, along with industry applications (Frank et al., 2009). The simplicity and user-friendliness of user interfaces is an important feature of Weka which makes it popular among researchers. 
Rattle: Rattle (the R Analytical Tool To Learn Easily) is a GUI package for R, primarily intended for the purpose of data mining. Rattle is used both as an instructional tool for data mining in universities and also by consultants and data scientists worldwide (Williams, 2009). Interactions with the interface are also recorded as scripts in the underlying $\mathrm{R}$ console, so that these scripts can be used independently of the GUI. Text mining capability has also been added to rattle in its later versions.

Orange: Orange is an open-source program for data mining, data visualization, and machine learning. Python scripting is used in the back-end. It provides an interactive visualization and analysis platform (Demšar et al., 2013). Operations are done through interactive workflows combined of widgets, which can be either custom-defined or predefined. Widgets for Bioinformatics, Time Series Analysis, Natural Language Processing and text mining etc. also available as add-ons in Orange, along with customary data mining capabilities.

KH Coder: $\mathrm{KH}$ Coder is a light-weight program which is developed specifically for quantitative content analysis and text mining. It was developed by Koichi Higuchi for personal research purpose and later offered as an open-source software under the GNU GPL license. Various text-mining procedures can be performed with $\mathrm{KH}$ Coder. An elaborate documentation is also available online.

\section{Conclusion}

Usage of software in the various stages of academic research is on the rise. Hence it is imperative that researchers pay more attention to the various issues and options in the area of research software, irrespective of their area of academic expertise. Early career researchers and students should have a keen interest in the various happenings in this area to stay updated in their academic pursuit. There is no claim that the list of software presented here is exhaustive or complete. It is hoped that this introduction to the software various packages 
available to the researchers in social science will inspire some of them to explore the hitherto unexplored areas, since research is also identified as a process of defying the beaten path to discover hidden knowledge.

\section{References}

Chambers, J. M. (2009). Facets of R. The R Journal, 1(1), 5-8.

Demšar, J., Curk, T., Erjavec, A., Gorup, Č., Hočevar, T., Milutinovič, M., Možina, M., Polajnar, M., Toplak, M., \& Starič, A. (2013). Orange: Data mining toolbox in Python. The Journal of Machine Learning Research, 14(1), 2349-2353.

Downie, T. (2016). Using the R Commander: A Point-and-Click Interface for R. Journal of Statistical Software, 75(Book Review 3). https://doi.org/10.18637/jss.v075.b03

Enright, J., \& Klippenstein, J. (2004). Tanagra: An Evaluation. University of Alberta. https://webdocs.cs.ualberta.ca/ zaiane/courses/cmput695-04/work/A2reports/tanagra.pdf

Fellows, I. (2012). Deducer: A data analysis GUI for R. Journal of Statistical Software, 49(8), 1-15.

Foucault, M. (1980). Power/knowledge: Selected interviews and other writings, 1972-1977. Pantheon.

Frank, E., Hall, M., Holmes, G., Kirkby, R., Pfahringer, B., Witten, I. H., \& Trigg, L. (2009). Weka-a machine learning workbench for data mining. In Data mining and knowledge discovery handbook (pp. 1269-1277). Springer.

GNU Project. (2015). GNU PSPP for GNU/Linux (0.8.5) [Computer software]. Free Software Foundation. https://www.gnu.org/software/pspp/

Hammer, Ø., Harper, D. A. T., \& Ryan, P. D. (2001). PAST: PALEONTOLOGICAL STATISTICS SOFTWARE PACKAGE FOR EDUCATION AND DATA ANALYSIS. Palaeontologia Electronica, 4(1), 1-9. 
Ihaka, R., \& Gentleman, R. (1996). R: A Language for Data Analysis and Graphics. Journal of Computational and Graphical Statistics, 5, 299-314.

Lamprianou, I. (2019). Applying the Rasch Model in Social Sciences Using R (1st edition). Routledge.

Miller, W. (2013). Statistics and Measurement Concepts with OpenStat. Springer-Verlag. //www.springer.com/in/book/9781461457428

Rakotomalala, R. (2005). TANAGRA: a free software for research and academic purposes. Proceedings of EGC'2005, 2, 697-702.

SOFA. (2017). SOFA Statistics (1.4.6) [Computer software]. Paton-Simpson \& Associates Ltd. https://www.sofastatistics.com

The jamovi project. (2020). Jamovi (1.2) [Computer software]. https://www.jamovi.org The JASP Team. (2015). Software to Sharpen Your Stats. APS Observer, 28(3). https://www.psychologicalscience.org/observer/bayes-or-bust-with-new-softwares Williams, G. J. (2009). Rattle: A Data Mining GUI for R. The R Journal, 1(2), 45-55.

About the author: Dr. Chinchu C. is a Consulting Psychologist, Podcaster, Writer, Educator, and Research Consultant from Kerala, India. His short bio is available at https://www.mhinnovation.net/profile/dr-chinchu-c

Sources of Funding: No funding agencies have provided financial support or funding for writing this article. 